\title{
Microglia-induced reactive astrocytes - toxic players in neurological disease?
}

Astrocytes can lose their normal protective features and take on a neurotoxic function that might play a part in CNS injury and neurodegeneration, according to a new study published in Nature. The team, led by Ben Barres, found that activated microglia induce the formation of harmful A1 astrocytes in areas that are vulnerable to cell death in several neurodegenerative disorders.

Astrocytes fulfil numerous crucial functions in the CNS, such as the provision of trophic support to neurons, clearance of debris, and modulation of synapse formation and function. In injury and disease, these cells can transform into 'reactive' astrocytes, which can be neuroprotective or neurotoxic in different circumstances. However, the function of reactive astrocytes in disease, and whether therapies could be targeted to these cells, remains unclear.

"For so many years, the focus of neurodegenerative disease treatment and research has mostly targeted

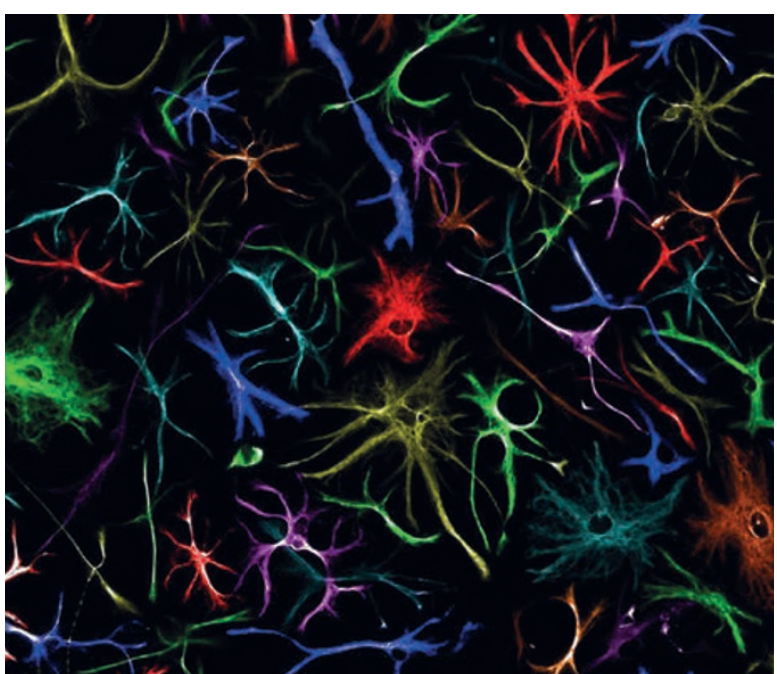

False-colour image of mouse astrocytes following activation with IL-1 $\alpha$, tumour necrosis factor and compliment factor $\mathrm{C} 1 \mathrm{q}$ in vitro. Image courtesy of S. A. Liddelow. neurons," remarks lead author of the new study, Shane Liddelow. "We are now learning of the important role that astrocytes and microglia play in neurodegeneration."

Barres and his team previously identified two classes of reactive astrocytes: A1 astrocytes, which they predicted to have a harmful role, and A2 astrocytes, which they predicted to have a protective role. In their new work, they set out to characterize the potentially destructive $\mathrm{A} 1$ astrocytes.

The researchers found that $\mathrm{A} 1$ astrocytes were generated in wildtype mice after injection of lipopolysaccharide (LPS), which activates microglia. However, LPS injection in Csf1r-knockout mice that lack microglia did not produce A1 astrocytes, suggesting that activated microglia induce A1 astrocytes.

Media conditioned by LPSactivated microglia in vitro, but not media from nonactivated microglia, also induced the A1 phenotype in cultured astrocytes. IL-1 $\alpha$, tumour necrosis factor (TNF) and compliment factor C1q were all upregulated after microglial activation, and the addition of neutralizing antibodies against these molecules to block their signalling prevented microgliaconditioned media from inducing the Al phenotype.

Barres and co-workers went on to show that A1 astrocytes lose their usual helpful functions and take on a toxic role. When cultured with A1 astrocytes, retinal ganglion cells (RGCs) made 50\% fewer connections than those grown with nonactivated astrocytes, and their remaining synaptic connections were weaker. In addition, $\mathrm{A} 1$ astrocytes had a decreased ability to phagocytose myelin debris and synaptosomes.
Importantly, media conditioned by $\mathrm{A} 1$ astrocytes was highly toxic to a variety of neuronal cells, including RGCs, cortical neurons, spinal motor neurons and mature oligodendrocytes. This action could not be blocked with antibodies against IL-1 $\alpha$, TNF or C1q, indicating the presence of an as-yet unknown toxic entity. Furthermore, crushing of the optic nerve in rats increased A1 astrocyte generation in the animals and was associated with the death of the injured neurons. However, this effect could be blocked by antibodies against IL-1a, TNF and C1q, which halted the generation of $\mathrm{A} 1$ astrocytes and delayed neuronal death.

Finally, Barres and colleagues investigated whether $\mathrm{A} 1$ astrocytes might be important in human neurodegenerative disease. Immunostaining for $\mathrm{C} 3$, a marker of A1 astrocytes, was positive in postmortem brain tissue from patients who had Alzheimer disease, Huntington disease, Parkinson disease, amyotrophic lateral sclerosis or multiple sclerosis. "What was most exciting and remarkable was that once we were able to visualize these reactive astrocytes, we saw that they were largely present in those brain regions associated with neurodegeneration" explains Liddelow.

The team think that these cells could represent an important target for future treatments. "Our results show that a broad range of brain disorders might be treatable by blocking astrocytes' metamorphosis into toxic cells, or by pharmaceutically countering the neuron-killing toxin that harmful astrocytes almost certainly secrete," concludes Liddelow. "We strongly believe that the development of more-effective drugs to treat astrocyte reactivity will help to treat a broad range of neurodegenerative diseases."

Charlotte Ridler

ORIGINAL ARTICLE Liddelow, S. A. et al. Neurotoxic reactive astrocytes are induced by activated microglia. Nature http://dx.doi. org/10.1038/nature21029 (2017) 